\title{
The Detection of Weak Signal Patterns in Radar Ocean Intensity Images
}

Roger Manasse

June 15, 1996

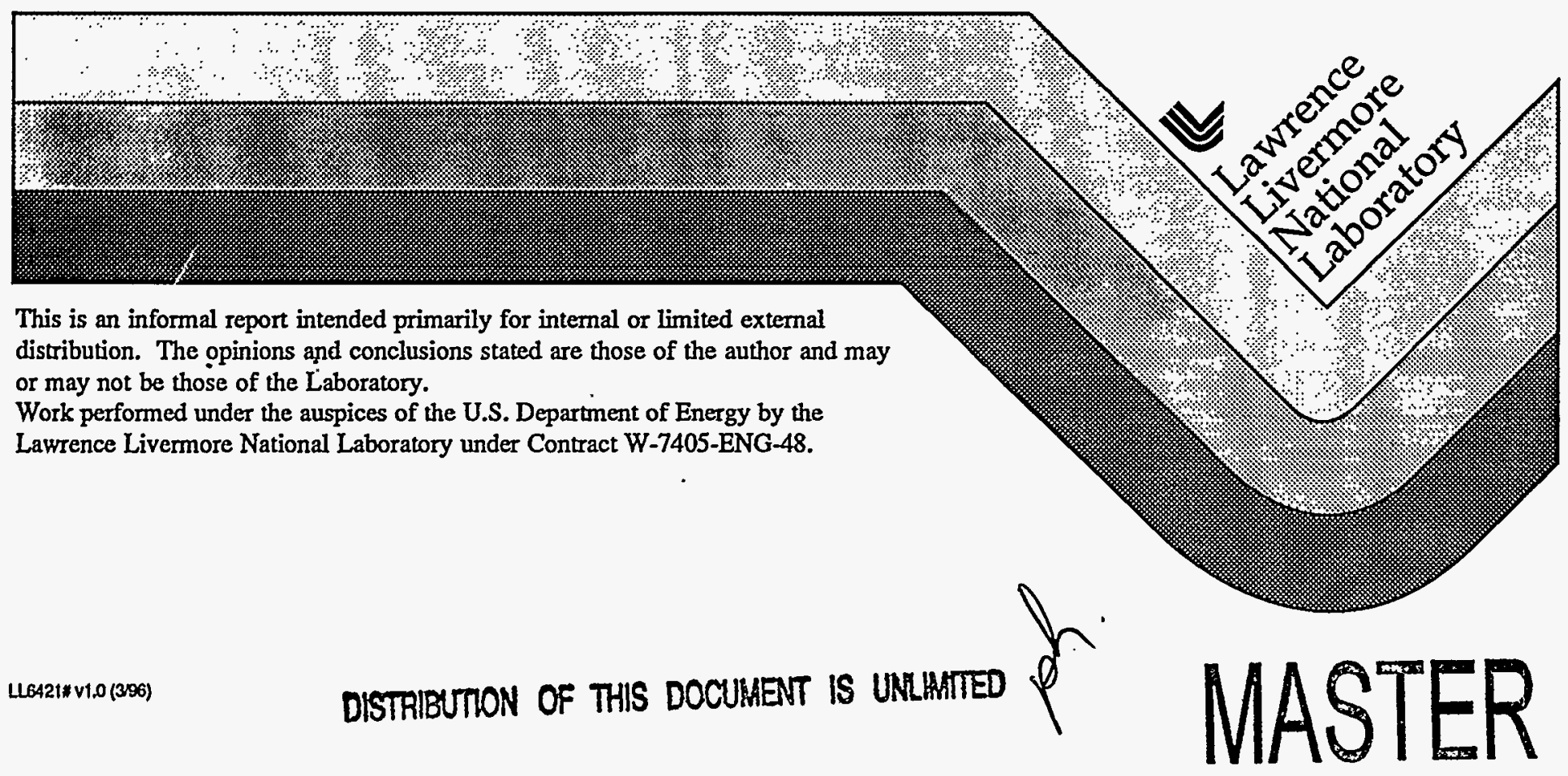




\section{DISCLAIMER}

This document was prepared as an account of work sponsored by an agency of the United States Government. Neither the United States Government nor the University of California nor any of their employees, makes any warranty, express or implied, or assumes any legal liability or responsibility for the accuracy, completeness, or usefulness of any information, apparatus, product, or process disclosed, or represents that its use would not infringe privately owned rights. Reference herein to any specific commercial product, process, or service by trade name, trademark, manufacturer, or otherwise, does not necessarily constitute or imply its endorsement, recommendation, or favoring by the United States Government or the University of California. The views and opinions of authors expressed herein do not necessarily state or reflect those of the United States Government or the University of California, and shall not be used for advertising or product endorsement purposes.

This report has been reproduced directly from the best available copy.

Available to DOE and DOE contractors from the Office of Scientific and Technical Information P.O. Box 62, Oak Ridge, TN 37831

Prices available from (615) 576-8401, FTS 626-8401

Available to the public from the National Technical Information Service

U.S. Department of Commerce 5285 Port Royal Rd. Springfield, VA 22161 


\section{DISCLAIMER}

Portions of this document may be illegible in electronic image products. Images are produced from the best available original document. 


\title{
THE DETECTION OF WEAK SIGNAL PATTERNS IN RADAR OCEAN INTENSITY IMAGES
}

\author{
ROGER MANASSE
}

JUNE 15, 1996

\begin{abstract}
The detection of weak patterns in radar ocean RCS images is complicated by the fact that signals and noise are interactive rather than additive, and the ambient noise background is non Gaussian, or even strongly non Gaussian at low grazing angles. This paper addresses this extremely difficult problem with the aid of two simplifying assumptions, 1) the signal modulation is weak and 2) the departure from Gaussianity is small. In situations where the departure from Gaussianity is large, an approach is suggested for reducing this non Gaussianity. The relevant weak signal detection theory, based on the Likelihood ratio, is reviewed and adapted for use in the analysis. The approach to this problem, similar to that previously used by the author for complex images, is facilitated by approximating the multivariate probability distributions as a composite integral involving underlying processes which are assumed to be Gaussian. This formulation, subject to the approximations in the analysis, permits the derivation of an ideal detection statistic (which determines the form of the optimum receiver) and a SNR which characterizes detection performance in the weak signal limit. Some implications for image processing are discussed and directions for future analysis are suggested
\end{abstract}




\title{
TABLE OF CONTENTS
}

\author{
Abstract \\ 1. Introduction \\ 2. The Relevant Detection Theory and lts Specialization \\ to Cases of Interest \\ 2.1 The General Likelihood Ratio Formulation \\ 2.2 The Generalized Signal-to-Noise Ratio \\ 2.3 The Weak Signal Limit \\ 2.4 Statistically Independent Samples Case \\ 2.5 The Additive Signal Case \\ 2.6 Interactive Signal and Noise Case \\ 2.7 The Multivariate Gaussian Noise Case \\ 2.7.1 A Non Ideal Detection Statistic \\ 2.7.2 Specialization to Whitened Noise statistics \\ 3. Specific Assumptions of the Detection Analys is \\ 4. The Weakly Non Gaussian Formulation \\ 4.1 Review of the Complex Image Formulation \\ 4.2 Transition to the Real Intensity Image \\ 4.3 The Ideal Detection statistic \\ 4.4 Derivation of the SNR \\ 5. An Approach for Handling Strongly Non Gaussian Statistics \\ 5.1 Introduction \\ 5.2 Processing of Single Pixel statistics \\ 5.2.1 The Under lying Gaussian Case \\ 5.2.2 Pixel statistics for LGA Sea Clutter \\ 5.2.3 The SNR Loss for K-Distributed sea Clutter \\ 5.2 .4 Restoration of the SNR Loss \\ 5.2.5 Transformation of Probability Distributions \\ 6. Implications for Processing Improvements and Further \\ Analysis \\ 6.1 Introduction \\ 6.2 Two-Dimensional Spatial Detrending \\ 6.3 Two-Dimensional Spectral Detrending \\ 6.4. Additional Processing Steps
}

Glossary of Symbols and Acronyms

References 


\section{Introduction}

The detection of signals of known form (i.e., including one or several unknown parameters) imbedded in additive Gaussian noise is a classic problem in detection theory for which the solution is well known, although not always computationally simple (see, for example $[1,2,3,4]$ ). The extension of this theory from the one dimensional case, i.e., a time waveform, to multiple dimensions, e.g., involving space and time coordinate variables, is straightforward and well understood.

During the intervening years since this theory was developed, more complicated detection problems have arisen where the underlying statistics for both signal and noise are more complex, and for which the determination of the optimum receiver and its performance are much more difficult. These complicating features may include one or more of the following:

- The signal is stochastic (rather than deterministic) and non stationary.

- The signal and noise are interactive (e.g., multiplicative) rather than additive

- The ambient noise is non Gaussian (perhaps strongly so)

- The ambient noise has cell-to-cell (i.e., pixel-to-pixel) statistical dependence which may extend out to substantial distances.

Occasionally, one can make simplifying assumptions such as a) the perturbation of the signal is weak and b) the non Gaussianity of the background noise is small, which facilitate the development of an approximate theory $[5,6]$.

The basic approach to this more complex problem using the Likelihood Ratio (LR) remains applicable. The different detection criteria (e.g., Neyman Pearson, I deal Observer, Bayes, Minimax) all lead to the LR criterion, the only difference being that the computed threshold on the LR is different [4]. As a practical matter, however, the solution of the more complicated detection problem noted above (i.e., the determination of the optimum receiver and its performance) is not tractable except in very special cases [6].

The specific application of interest here is the detection of modulation patterns in high resolution (either RAR or SAR) radar images of the ocean surface. Such patterns could be caused by a variety of phenomena. One example is the modulation of surface waves by surface currents due to tidal flows over regions of variable depth, or those due to naturally occurring or artificially induced internal waves. These patterns can be weak, but may extend over an area large compared to resolution cell dimensions. 
The radar ocean imaging problem of interest will permit the use of certain simplifying assumptions. An exact solution of this detection problem is still elusive, however, and it will be necessary to rely heavily on heuristic methods, for the most part. Nevertheless, the writer believes that the analysis will shed considerable light on the image processing which is needed for enhancing the visibility of ocean surface features of interest.

Although some of the results presented in this paper may be new, the writer makes no claim for originality as he has not conducted an exhaustive survey of the detection literature.

The next section reviews some relevant fundamental detection theory based on the use of the Likelihood Ratio. 
2. Relevant Detection Theory and its Specialization to Cases of Interest

\subsection{The General Likel ihood Ratio Formulation}

Let the received information be in the form of a column vector $Z=\left[Z_{i}\right], i=1,2, \ldots, N$, where the $Z_{i}$ may be real or complex. The $i$ may represent a concatenated index which denotes cell or pixel location in a multidimensional image (e.g., space-space or space-time). In general the $Z_{i}$ are statistically dependent and non Gaussian. The LR, $L(z)$, is a multivariate function which is the ratio of two probability densities.

$$
\mathrm{L}(\mathrm{Z})=\frac{\mathrm{P}_{\mathrm{SN}}(\mathrm{Z})}{\mathrm{P}_{\mathrm{N}}(\mathrm{Z})}
$$

where the subscripts $S N$ indicate that both signal and noise are present and $N$ indicates that only noise is present.

Essentially all optimum binary decision rules involve setting a simple threshold on $L(Z)$ to decide on the presence or absence of a signal. The only difference between these detectors is the value of the threshold which is employed.

$P_{S N}$, and thus $I$, may include the presence of a parameter space of signals with its own a priori probability distribution. This leads to the use of a conditional LR, L(Z/ $\gamma)$, where $\gamma=\left[\gamma_{i}\right]$ is a set of parameters which define the signal. This formulation is well known in the theory of detection and parameter estimation, but will not be discussed further here. The existence of a signal parameter space does not detract from the utility of the LR in most cases of interest.

One can write

$$
\mathrm{P}_{\mathrm{SN}}(\mathrm{Z})=\mathrm{P}_{\mathrm{N}}(\mathrm{Z})+\delta \mathrm{P}_{\mathrm{N}}(\mathrm{Z})
$$

which defines the function $\delta P_{N}(Z)$. Since $P_{S N}(Z)$ and $P_{N}(Z)$ each integrate to unity, one must have

$$
\int \delta \mathrm{P}_{\mathrm{N}}(\mathrm{Z}) \mathrm{dZ}=0
$$

Recall the $Z$ here is a vector, so that the above integral is actual1y $\mathrm{N}$-dimensional, i.e.,

$$
\int 0 \mathrm{dz}=\int \ldots \int 0 \mathrm{dz} \mathrm{z}_{1} d \mathrm{z}_{2} \ldots d \mathrm{z}_{\mathrm{N}}
$$

Then $L(Z)$ is, from $(2-1)$ 


$$
\mathrm{L}(\mathrm{Z})=1+\frac{\delta \mathrm{P}_{\mathrm{M}}(\mathrm{Z})}{\mathrm{P}_{\mathrm{N}}(\mathrm{Z})}
$$

An ideal statistic, denoted $\chi$, is defined as any function which is monotonically related to $L(z)$. For the purpose of making a decision on the presence or absence of a signal, setting a threshold on $\chi$ is then equivalent to setting a threshold on $L(z)$. Examples of ideal statistics are

$$
\begin{aligned}
& \chi=\ln L(Z) \\
& \chi=\frac{\delta \mathrm{P}_{\mathrm{N}}(\mathrm{Z})}{\mathrm{P}_{\mathrm{N}}(\mathrm{Z})}
\end{aligned}
$$

Generally the first statistic, $\ln L(\mathrm{z})$, is preferred in the weak signal case because its statistics often become Gaussian, by virtue of the Central Limit Theorem (CLT).

\subsection{The Generalized Signal-to-Noise Ratio}

The generalized SNR, sometimes referred to as the deflection criterion, is defined as (see p. 308 of [7])

$$
\operatorname{SNR}=\frac{\left[\langle\chi\rangle_{\mathrm{SN}}-\langle\chi\rangle_{\mathrm{N}}\right]^{2}}{\left\langle\chi^{2}>_{\mathrm{N}}-\langle\chi\rangle_{N}^{2}\right.}
$$

Here <> denotes expectation and the subscripts denote where noise alone or signal and noise are present. The denominator will be recognized as the variance of $\chi$. In general this SNR does not uniquely characterize detection performance (in terms of a ROC curve, $P_{D}$ vs. $P_{F}$ ). However, in the important situation where $\chi$ has Gaussian statistics, e.g., by virtue of the CLT, and the variance of $\chi$ is essentially unchanged by the presence of the signal, e.g., because the signal is weak, this SNR completely characterizes detection performance, and standard detection curves can be employed for $P_{D}$ vs. $P_{F}$.

The SNR is readily shown to be invariant to an affine transformation [8] on $\chi$, namely

$$
\chi \rightarrow a x+b
$$

where $a$ and $b$ are arbitrary constants. This transformation both preserves $\chi$ as an ideal statistic and SNR. In particular, it is always possible to choose $a$ and $b$ so that $\langle\chi\rangle_{N}=0$, and the SNR expression simplifies accordingly.

Now compute SNR for the case where one chooses $\chi=L(Z)$. The required quantities are then 


$$
\begin{aligned}
& <\chi>_{N}=\int \mathrm{L}(\mathrm{Z}) \mathrm{P}_{\mathrm{N}}(\mathrm{Z}) d \mathrm{Z}=1 \\
& <\chi>_{\mathrm{SN}}=\int \mathrm{L}(\mathrm{Z})\left[\mathrm{P}_{\mathrm{N}}(\mathrm{Z})+\delta \mathrm{P}_{\mathrm{N}}(\mathrm{Z})\right] \mathrm{dZ}=1+\int \frac{\left[\delta \mathrm{P}_{\mathrm{NN}}(\mathrm{Z})\right]^{2}}{\mathrm{P}_{\mathrm{N}}(\mathrm{Z})} \mathrm{dZ} \\
& <\chi^{2}>_{\mathrm{N}}=\int \mathrm{L}^{2}(\mathrm{Z}) \mathrm{P}_{\mathrm{N}}(\mathrm{Z}) \mathrm{dZ}=1+\int \frac{\left[\delta \mathrm{P}_{\mathrm{N}}(\mathrm{Z})\right]^{2}}{\mathrm{P}_{\mathrm{N}}(\mathrm{Z})} \mathrm{dZ} .
\end{aligned}
$$

Inserting these into (2-7) yields for the SNR

$$
\mathrm{SNR}=\int \frac{\left[\delta \mathrm{P}_{\mathrm{N}}(\mathrm{Z})\right]^{2}}{\mathrm{P}_{\mathrm{N}}(\mathrm{Z})} d \mathrm{Z}
$$

No approximations have been made in deriving this formula.

It is of interest to know whether the choice $\chi=L(z)$, or more generally $a L(z)+b$, actually maximizes the SNR. Without loss of generality, one can assume that $\chi$ is translated so that $\langle\chi\rangle_{\mathrm{N}}=0$, in which case $(2-7)$ can be written

$$
\operatorname{SNR}=\frac{\left(\int \chi(\mathrm{Z}) \delta \mathrm{P}_{\mathrm{N}}(\mathrm{Z}) \mathrm{dz}\right)^{2}}{\int \chi^{2}(\mathrm{Z}) \mathrm{P}_{\mathrm{N}}(\mathrm{Z}) \mathrm{dz}}
$$

The N-dimensional extension of Schwartz's inequality [9] applied to the numerator yields

$$
\left(\int \chi(\mathrm{Z}) \delta \mathrm{P}_{\mathrm{N}}(\mathrm{Z}) d \mathrm{Z}\right)^{2} \leq \int \chi^{2}(\mathrm{Z}) \mathrm{P}_{\mathrm{N}}(\mathrm{Z}) d \mathrm{Z} \int \frac{\left[\delta \mathrm{P}_{\mathrm{N}}(\mathrm{Z})\right]^{2}}{\mathrm{P}_{\mathrm{N}}(\mathrm{Z})} \mathrm{dZ}
$$

Thus,

$$
\mathrm{SNR} \leq \int \frac{\left[\delta \mathrm{P}_{\mathrm{N}}(\mathrm{Z})\right]^{2}}{\mathrm{P}_{\mathrm{N}}(\mathrm{Z})} \mathrm{dZ}
$$

The equality is obeyed if and only if

$$
\chi \sim \delta \mathrm{P}_{\mathrm{N}}(\mathrm{Z}) / \mathrm{P}_{\mathrm{N}}(\mathrm{Z})=\mathrm{L}(\mathrm{Z})-1
$$

Thus, this important result states that the detection statistic must be related to $L(Z)$ by an affine transformation in order to achieve a maximum SNR. 


\subsection{The Weak Signal Limit}

The above formulas readily specialize to the single pixel case simply by setting $z=z_{1}$. Eq. $(2-2)$ is then

$$
\mathrm{P}_{\mathrm{SN}}\left(\mathrm{Z}_{\mathrm{i}}\right)=\mathrm{P}_{\mathrm{N}}\left(\mathrm{Z}_{\mathrm{i}}\right)+\delta \mathrm{P}_{\mathrm{N}}\left(\mathrm{Z}_{\mathrm{i}}\right)
$$

In the weak signal case it is often possible (under appropriate conditions) to approximate

$$
\delta \mathrm{P}_{\mathrm{N}}\left(\mathrm{Z}_{\mathrm{i}}\right)<<\mathrm{P}_{\mathrm{N}}\left(\mathrm{Z}_{\mathrm{i}}\right)
$$

at least in the region where $\mathrm{P}_{N}\left(\mathrm{Z}_{i}\right)$ has significant value. The ever-present receiver noise assists in the validity of this approximation. Then

$$
L_{i}\left(Z_{i}\right) \equiv \frac{P_{S N}\left(Z_{i}\right)}{P_{N}\left(Z_{i}\right)}=1+\frac{\delta P_{N}\left(Z_{i}\right)}{P_{N}\left(Z_{i}\right)}
$$

and one can approximate

$$
\operatorname{In} I_{i}\left(Z_{i}\right)=\delta \operatorname{In} P_{N}\left(Z_{i}\right) \cong \frac{\delta P_{N}\left(Z_{i}\right)}{P_{N}\left(Z_{i}\right)}<1
$$

Thus, to this degree of approximation, $\ln I_{i}\left(Z_{i}\right)$ is an ideal statistic which maximizes SNR, Denoted $S N R_{i}$, for the ith pixel.

Furthermore, let $Y_{i}(x)$ be any arbitrary but well behaved monotonic function of its argument, e.g., the nth power. Then

$$
\begin{aligned}
Y_{i} & \equiv Y_{i}\left(\operatorname{InL} L_{i}\left(Z_{i}\right)\right) \\
& \cong Y_{i}(0)+Y_{i}^{\prime}(0) \ln L_{i}\left(Z_{i}\right) \\
& \cong Y_{i}(0)+Y_{i}^{\prime}(0) \frac{\delta P_{N}\left(Z_{i}\right)}{P_{N}\left(Z_{i}\right)}
\end{aligned}
$$

where higher order terms have been neglected. It is seen that $Y_{i}$ is also an ideal statistic which maximizes SNR $i$ [10].

Combining $(2-10)$ and $(2-18)$, one can obtain the following alternative approximate expressions for SNR; which are sometimes useful.

$$
\begin{aligned}
\operatorname{SNR}_{i} & \cong \int\left(\operatorname{In} L_{i}\left(Z_{i}\right)\right)^{2} P_{N}\left(Z_{i}\right) d Z_{i} \\
& \cong \int\left(\delta \operatorname{In} P_{N}\left(Z_{i}\right)\right)^{2} P_{N}\left(Z_{i}\right) d Z_{i}
\end{aligned}
$$




\subsection{Case of Statistically Independent Samples}

Consider the important special case where the $\mathbf{Z}_{i}$ for each sample (or pixel) is statistically independent of the others. Then $P_{1}(Z), P_{S N}(Z)$, and $I(Z)$ factor into a product of independent functions. $I(Z)$ is then seen to be a product of the $I_{i}\left(Z_{i}\right)$ for the independent samples.

$$
L(\mathrm{Z})=\prod_{i} L_{i}\left(\mathrm{Z}_{i}\right)
$$

We choose for the ideal detection statistic

$$
\chi=\ln I(\mathrm{Z})=\sum_{i} \ln L_{i}\left(Z_{i}\right)
$$

This choice for $\chi$ is desirable because the probability density for $\chi$ will tend to be Gaussian for a weak signal which is distributed across many pixels, by virtue of the CLT.

It readily follows, using $(2-18)$ and $(2-3)$, that

$$
\begin{aligned}
& \langle\chi\rangle_{N}=\sum_{i}\left\langle\operatorname{In} I_{i}\left(Z_{i}\right)\right\rangle_{N} \cong 0 \\
& \left.\langle\dot{\chi}\rangle_{\mathrm{SN}}=\sum_{i}<\operatorname{In} L_{i}\left(\mathrm{Z}_{i}\right)\right\rangle_{\mathrm{SN}} \cong \sum_{i} \int \frac{\left[\delta \mathrm{P}_{\mathrm{N}}\left(\mathrm{Z}_{i}\right)\right]^{2}}{\mathrm{P}_{\mathrm{N}}\left(\mathrm{Z}_{i}\right)} \mathrm{dZ} \mathrm{Z}_{\mathrm{i}} \\
& \left\langle\chi^{2}>_{N}=\sum_{i} \int\left[\ln L_{i}\left(Z_{i}\right)\right]^{2} P_{N}\left(Z_{i}\right) d Z_{i} \cong \sum_{i} \int \frac{\left[\delta \mathrm{P}_{N}\left(Z_{i}\right)\right]^{2}}{\mathrm{P}_{N}\left(Z_{i}\right)} d Z_{i}\right.
\end{aligned}
$$

Insertion of these into the generalized SNR formula (2-7) yields

$$
S N R=\sum_{i} \int \frac{\left[\delta \mathrm{P}_{\mathrm{N}}\left(\mathrm{Z}_{i}\right)\right]^{2}}{\mathrm{P}_{\mathrm{N}}\left(\mathrm{Z}_{\mathrm{i}}\right)} \mathrm{d} \mathrm{Z}_{\mathrm{i}}
$$

SNR $i$ for the ith cell is obtained by setting $z=z_{i}$ in $(2-10)$, whence

$$
\operatorname{SNR}=\sum_{i} \operatorname{SNR}_{i}
$$

a result which is not unexpected. It is not hard to show that this relation applies generally for an optimized weighted sum of independent random variables (see the Appendix of [10], for examp le).

Even though SNR $i$ may not characterize the detectability for the individual $z_{i}$, SNR will characterize detectability if the detection statistic has become Gaussian by virtue of the CLT, and the presence of the weak signal does not significantly change the 
variance of the noise. SNR; has important significance in the weak signal case because it represents the contribution of the ith cell to overall detection performance given by SNR. Any single cell processing, e.g., a zero memory nonlinear (ZMNL) function, which increases SNR $i$ will increase its contribution to SNR.

The assumption of statistical independence can sometimes be useful in a situation where it is not strictly obeyed. For example, if the statistics of $z i$ are a slowly varying function of $i$, i.e., the location of the ith cell in an image, it may be permissible to assume statistical independence on a local basis for the purpose of performing optimized local averages. If the local averages contain a reasonably large number of pixels, the resulting statistics would tend to be Gaussian by virtue of the CLT. This heuristic view provides some justification for spatial-temporal filtering, particularly where signal modulations change very slowly with respect to pixel dimensions.

\subsection{The Additive Signal Case}

Thus far it has been assumed that the perturbation of the $\mathrm{Zi}$ due to the presence of a signal is arbitrary. Consider the special case where the perturbation is an additive signal $\Delta \mathrm{z}_{i}$. Then

$$
\mathrm{P}_{S N}\left(\mathrm{Z}_{i}\right)=\mathrm{P}_{N}\left(\mathrm{Z}_{i}-\Delta \mathrm{Z}_{i}\right) \cong \mathrm{P}_{N}\left(\mathrm{Z}_{i}\right)-\frac{d \mathrm{P}_{\mathrm{N}}\left(\mathrm{Z}_{\mathrm{i}}\right)}{d \mathrm{z}_{i}} \Delta \mathrm{Z}_{i}
$$

and

$$
\begin{aligned}
\frac{\delta \mathrm{P}_{N}\left(\mathrm{Z}_{i}\right)}{\mathrm{P}_{\mathrm{N}}\left(\mathrm{Z}_{i}\right)} & \cong \frac{1}{\mathrm{P}_{N}\left(\mathrm{Z}_{i}\right)} \frac{d \mathrm{P}_{\mathrm{N}}\left(\mathrm{Z}_{i}\right)}{d \mathrm{Z}_{i}} \Delta \mathrm{Z}_{i} \\
& =-\frac{\mathrm{d}}{d \mathrm{Z}_{i}}\left(\ln \mathrm{P}_{\mathrm{N}}\left(\mathrm{Z}_{i}\right)\right) \Delta \mathrm{Z}_{i}
\end{aligned}
$$

Recalling (2-10) for the SNR, one then has

$$
\operatorname{SNR}_{i} \cong\left(\Delta \mathrm{Z}_{i}\right)^{2} \int\left[\frac{\mathrm{d}}{\mathrm{dz}}\left(\operatorname{In} \mathrm{P}_{\mathrm{N}}\left(\mathrm{Z}_{i}\right)\right)\right]^{2} \mathrm{P}_{\mathrm{N}}\left(\mathrm{Z}_{i}\right) d \mathrm{Z}_{i}
$$

This expression will be recognized as the single pixel SNR obtained by Middleton [11]. The additive signal assumption is valid for many important detection problems. Unfortunately, it is not valid for the detection of ocean surface features of interest, but this case is included here for the sake of completeness. 


\subsection{Interactive Signa1 and Noise}

Consider the case where the signal is not simply additive, but interactive. For example, the signal could perturb the statistics of the ambient background. In the case of Gaussian noise, for example, these statistics would be represented by a moment matrix $M$. For the noise only case one can write

$$
\mathrm{P}_{\mathrm{N}}(\mathrm{Z})=\mathrm{P}(\mathrm{Z} / \mathrm{M})
$$

When signal is present

$$
\mathrm{P}_{\mathrm{SN}}^{\prime}(\mathrm{Z})=\mathrm{P}(\mathrm{Z} / \mathrm{M}+\delta \mathrm{M})=\mathrm{P}_{\mathrm{N}}(\mathrm{Z})+\delta \mathrm{P}_{\mathrm{N}}(\mathrm{Z})
$$

where $\delta M=\left[\delta M_{i j}\right]$ is the perturbation of $M$ due to the presence of signal, and $\delta M \ll M$. The above $P_{N}(Z)$ and $\delta P_{N}(Z)$ are used in $(2-10)$ to compute SNR.

Thus it is seen that an interactive signal and noise model is readily handled with the basic detection formalism described above and it circumvents the need for the additive signal assumption.

In the very weak signal case one can (under appropriate conditions which are not further addressed here) assume

$$
\delta P_{N}(Z)<P_{N}(Z)
$$

in which case

$$
\frac{\delta \mathrm{P}_{\mathrm{N}}(\mathrm{Z})}{\mathrm{P}_{\mathrm{N}}(\mathrm{Z})} \cong \operatorname{In} \mathrm{L}(\mathrm{Z})=\delta \ln \mathrm{P}_{\mathrm{N}}(\mathrm{Z})
$$

To this approximation, all of these quantities are ideal

detection statistics which yield the maximum SNR. Insertion of $(2-32)$ "in the SNR formula $(2-10)$ yields

$$
S N R \cong \int\left(\delta \ln P_{N}(Z)\right)^{2} P_{N}(Z) d Z
$$

This approximate expression is often convenient for computing SNR.

2.7 The Multivariate Gaussian Noise Example

A case of frequent interest is the perturbation of mean zero Gaussian noise.

$$
P_{N}(Z)=P(Z / M)=\frac{1}{\sqrt{(2 \pi)^{N}|M|}} \exp \left(-\frac{1}{2} Z^{T} M^{-1} Z\right)
$$


where the moment matrix $M$ is given by

$$
M=\left[M_{i j}\right]=\left[<Z_{i} Z_{j}>\right]
$$

Then

$$
\delta \ln \mathrm{P}(\mathrm{Z} / \mathrm{M})=-\frac{1}{2} \delta \ln |\mathrm{M}|-\frac{1}{2} \mathrm{Z}^{\mathrm{T}} \delta \mathrm{M}^{-1} \mathrm{Z}
$$

This expression is simplified by using the following matrix relationships (see p. 97, 106 of [12]).

$$
\begin{aligned}
\delta \operatorname{In}|M| & =\frac{1}{|M|}\{|M+\delta M|-|M|\}=\left|I_{N}+M^{-1} \delta M\right|-1 \\
& \cong \operatorname{Tr}\left(M^{-1} \delta M\right)
\end{aligned}
$$

where $\operatorname{Tr}\left(\right.$ ) stands for trace of a matrix and $I_{N}$ is the $N \times N$ identity matrix. Also

$$
\delta M^{-1} \cong-M^{-1} \delta M^{-1}
$$

Use of $(2-37)$ and $(2-38)$ in $(2-36)$ yields

$$
\delta \cdot \ln \mathrm{P}(\mathrm{Z} / \mathrm{M}) \cong-\frac{1}{2} \operatorname{Tr}\left(\mathrm{M}^{-1} \delta \mathrm{M}\right)+\frac{1}{2} \mathrm{Z}^{\mathrm{T}} \mathrm{M}^{-1} \delta \mathrm{MM}^{-1} \mathrm{Z}
$$

It is seen from (2-39) that an ideal detection statistic is simply

$$
\chi \cong \mathrm{Z}^{\mathrm{T}} \mathrm{M}^{-1} \delta \mathrm{MM}^{-1} \mathrm{Z}
$$

In order to calculate SNR, insert (2-39) into (2-33).

$$
\begin{gathered}
\mathrm{SNR}=\frac{1}{4}\left\{\operatorname{Tr}\left(\mathrm{M}^{-1} \delta \mathrm{M}\right)\right\}^{2}-\frac{1}{2} \operatorname{Tr}\left(\mathrm{M}^{-1} \delta \mathrm{M}\right)<\mathrm{Z}^{\mathrm{T}} \mathrm{M}^{-1} \delta \mathrm{MM}^{-1} \mathrm{Z}> \\
+\frac{1}{4}<\mathrm{Z}^{\mathrm{T}} \mathrm{M}^{-1} \delta \mathrm{MM}^{-1} \mathrm{ZZ}^{\mathrm{T}} \mathrm{M}^{-1} \delta \mathrm{MM}^{-1} \mathrm{Z}>
\end{gathered}
$$

where <> here indicates that the quantity is averaged over the ambient noise background. The first $\langle>$ in $(2-41)$ can be simplified to

$$
\begin{aligned}
<\mathrm{Z}^{\mathrm{T}} \mathrm{M}^{-1} \delta \mathrm{MM}^{-1} \mathrm{Z}> & =<\operatorname{Tr}\left(\mathrm{Z}^{\mathrm{T}} \mathrm{M}^{-1} \delta M M^{-1} \mathrm{Z}\right)> \\
& =\operatorname{Tr}\left(\mathrm{M}^{-1} \delta M^{-1}<\mathrm{Z}^{\mathrm{T}}>\right) \\
& =\operatorname{Tr}\left(\mathrm{M}^{-1} \delta \mathrm{M}\right)
\end{aligned}
$$

The second $\langle>$ in $(2-41)$ can be written 


$$
\begin{aligned}
& <\mathrm{Z}_{i}\left(M^{-1} \delta M M^{-1}\right)_{i j} Z_{j} Z_{k}\left(M^{-1} \delta M M^{-1}\right)_{k I} Z_{I}> \\
& \quad=\left(M^{-1} \delta M M^{-1}\right)_{i j}\left(M^{-1} \delta M M^{-1}\right)_{k l}<Z_{i} Z_{j} Z_{k} Z_{I}>
\end{aligned}
$$

where, according to the summation convention, there is an implicit sum over repeated indices. Use the product decomposition rule for real Gaussian variables to obtain,

$$
<\mathrm{Z}_{\mathrm{i}} \mathrm{Z}_{\mathrm{j}} \mathrm{Z}_{\mathrm{k}} \mathrm{Z}_{1}>=\mathrm{M}_{\mathrm{i} j} \mathrm{M}_{\mathrm{k} l}+\mathrm{M}_{\mathrm{ik}} \mathrm{M}_{\mathrm{jl}}+\mathrm{M}_{\mathrm{il}} \mathrm{M}_{\mathrm{jk}}
$$

Insert (2-44) in (2-43). Rearranging terms, one has for (2-43),

$$
<>=2 \operatorname{Tr}\left(\mathrm{M}^{-1} \delta \mathrm{MM}^{-1} \delta \mathrm{M}\right)+\left(\operatorname{Tr}\left(\mathrm{M}^{-1} \delta \mathrm{M}\right)\right)^{2}
$$

Inserting (2-45) and (2-42) into (2-41) and collecting terms, it readily follows that

$$
S N R=\frac{1}{2} \operatorname{Tr}\left(M^{-1} \delta M M^{-1} \delta M\right)
$$

This result is apparently well known. A very similar result can be derived for the complex multivariate Gaussian process, the only difference being the constant appearing in front of $\operatorname{Tr}()$, [3].

\subsubsection{A Non Ideal Detection Statistic}

Ideal or optimum detection statistics require a priori knowledge of the signal. Occasionally it is desirable to use a non optimum detection statistic which requires no a priori knowledge of the signal. One such statistic is the "whitening" filter employed by Novak and his coworkers at MIT $[13,14,15]$. The use of whitening filters has also been examined by $\mathrm{Dr}$. D. Holliday and his coworkers at RDA-Logicon and Dr. J. Brase at LLNL [16]. The non optimum detection statistic is

$$
\chi=\mathrm{Z}^{\mathrm{T}} \mathrm{M}^{-1} \mathrm{Z}
$$

The derivation of the SNR from the statistics of $\chi$ employs methods analogous to those used earlier, but the details are omitted here. The result is simply

$$
\operatorname{SNR}=\frac{1}{2 \mathrm{~N}}\left(\operatorname{Tr}\left(\mathrm{M}^{-1} \delta \mathrm{M}\right)\right)^{2}
$$

The SNR available from this non optimum statistic is never greater than that available from an optimum statistic, as one would expect. 


\subsubsection{Specialization to Whitened Noise Statistics}

The moment matrix $M$ of $Z$ may be known a priori, or it can be measured. Since $M$ is positive definite, the matrices $M^{1 / 2}$ and $M^{-1 / 2}$ are also positive definite and can be uniquely determined from $M$. It is then possible to transform to a new measurement vector $Y$ defined by

$$
\mathrm{Y} \equiv \mathrm{M}^{-1 / 2} \mathrm{Z}
$$

where $Y$ has whitened noise statistics. The moment matrix of $Y$, denoted $M_{y}$, is

$$
\left.\mathrm{M}_{Y}=<\mathrm{YY}^{\mathrm{T}}\right\rangle=\mathrm{M}^{-\frac{1}{2}}<\mathrm{ZZ}^{\mathrm{T}}>\mathrm{M}^{-\frac{1}{2}}=\mathrm{I}_{\mathrm{N}}
$$

The perturbation in $M_{y}$ due to the presence of a signal is

$$
\delta M_{y}=M^{-\frac{1}{2}} \delta M M^{-\frac{1}{2}}
$$

The optimum detection statistic of (2-40) can now be written with the aid of $(2-49)$ and $(2-51)$

$$
\begin{aligned}
\chi & =\mathrm{Z}^{\mathrm{T}^{-\frac{1}{2}}}\left(\mathrm{M}^{-\frac{1}{2}} \delta \mathrm{MM}^{-\frac{1}{2}}\right) \mathrm{M}^{-\frac{1}{2}} \mathrm{Z} \\
& =\mathrm{Y}^{\mathrm{T}} \delta \mathrm{M}_{\mathrm{Y}} \mathrm{Y}
\end{aligned}
$$

Equation (2-46) for the SNR can be written, using (2-51)

$$
\begin{aligned}
\text { SNR } & =\frac{1}{2} \operatorname{Tr}\left(M^{-\frac{1}{2}} \delta M^{-\frac{1}{2}} M^{-\frac{1}{2}} \delta M M^{-\frac{1}{2}}\right) \\
& =\frac{1}{2} \operatorname{Tr}\left(\left(\delta M_{Y}\right)^{2}\right)
\end{aligned}
$$

These expressions for $\chi$ and SNR have been simplified considerably by the whitening process.

One can also develop simplified formulas for the non optimum detection statistic, $(2-47)$, discussed in the previous section. With the whitening transformation $(2-49),(2-47)$ becomes

$$
\chi=\mathrm{Z}^{\mathrm{T}} \mathrm{M}^{-\frac{1}{2}} \mathrm{M}^{-\frac{1}{2}} \mathrm{Z}=\mathrm{Y}^{\mathrm{T}} \mathrm{Y}
$$

This $\chi$ is seen to reduce to a simple energy detector. The SNR expression, $(2-48)$, simplifies to

$$
\begin{aligned}
\text { SNR } & =\frac{1}{2 N}\left(\operatorname{Tr}\left(M^{-\frac{1}{2}} \delta M^{-\frac{1}{2}}\right)\right)^{2} \\
& =\frac{1}{2 N}\left(\operatorname{Tr}\left(\delta M_{Y}\right)\right)^{2}
\end{aligned}
$$


The use of a whitening filter not only simplifies the detection statistic and SNR formulas, it serves as a useful conditioning technique in the signal processing to prepare images for $v$ isual inspection. 


\section{Specific Assumptions of the Detection Analysis}

The following specific assumptions, which go beyond the general assumptions outlined in the introduction, will be invoked at appropriate points in the analysis which follows. These are, in no special order:

- The data is provided in the form of an image, e.g., two space dimensions, or one space and one time dimension.

- The cell (or pixel) outputs to be processed are a set of real, non negative intensities, $Z_{i}$, where $i$ is a concatenated index that denotes cell location and the $Z_{i}$ can be assembled into a vector $Z=\left[Z_{i}\right]$, where $i=1,2, \ldots, N$.

- The $Z_{i}$, which are formed by taking the square of the modulus of the cell outputs, are generally non Gaussian and have statistical dependence. (The complex cell outputs, from a RAR or SAR, which are used to calculate the $Z_{i}$, do contain phase. However, it will be assumed that this phase is random from cell to cell, contains no useful signal information, and thus may be discarded. The complex cell outputs are statistically uncorrelated but not statistically independent, in general).

- The ambient noise background is homogeneous and stationary (if a time coordinate is involved).

- The signal perturbation of ambient statistics, which is assumed to be weak, is generally neither homogeneous nor stationary.

- Sources of noise caused by radar artifacts or highly directional surface features such as swell waves or wave groups are ignored (techniques for mitigating these sources of noise are addressed elsewhere, although some of the techniques discussed in section 6 are relevant). 


\section{The Weakly Non Gaussian Formulation}

\section{1 Review of the Complex Image Formulation}

The starting point for the analysis is the detection formulation for complex images presented in [10] in which the signal causes a small perturbation of the ambient weakly non Gaussian noise statistics. $E=\left[E_{i}\right], i=1,2, \ldots N$, is the complex set of pixel outputs from an image, where $i$ is a concatenated index which, as before, denotes pixel location in space and/or time coordinates. The probability density for the vector $E$ is given by a conditional probability density. In the ambient noise case

$$
\mathrm{P}(\mathrm{E} / \mathrm{M}, \mathrm{Q})=\int \ldots \int \mathrm{P}(\mathrm{E} / \mathrm{C}) \mathrm{P}(\mathrm{C} / \mathrm{M}, \mathrm{Q}) \mathrm{dC}
$$

where

$$
\begin{aligned}
& P(E / C)=\frac{1}{(2 \pi)^{\mathbb{N}|C|}} \exp \left\{-\frac{1}{2} E^{+} C^{-1} E\right\} \\
& P(C / M, Q)=\frac{1}{(2 \pi)^{N^{2}|Q|}} \exp \left\{-\frac{1}{2}(C-M)^{+} Q^{-1}(C-M)\right\}
\end{aligned}
$$

are complex multivariate Gaussian distributions.

$$
\left.\mathrm{C}=\frac{1}{2}<\mathrm{EE}^{+}\right\rangle=\left[\mathrm{C}_{i j}\right]
$$

and

$$
Q=\frac{1}{2}<(C-M)(C-M)^{+}>=\left[Q_{i j k l}\right]
$$

where < > stands for an average with respect to the respective probability densities. $C$ is characterized by two subscripts, and hence has the form of a matrix, while $Q$ is characterized by four subscripts. $C$ is a dummy variable of integration and the integral over $c$ is multidimensional over all $C_{i j}$. Note that while $C$ has $N^{2}$ elements, $Q$ has $N^{4}$ elements. Symmetries will, of course, reduce the number of independent elements.

In the presence of a signal, the only change in $P(E / M, Q)$ is to replace $M$ by $M+\delta M$, where $\delta M \ll M$ is the perturbation produced by the signal.

The above expressions were used to determine approximate expressions for an ideal statistic and SNR, but these will not be repeated here. Note that all the relevant statistics are determined by the quantities $M$ and $Q$. 


\subsection{Transition to the Real Intensity Image}

Consider now the situation where the cross correlation of complex pixel outputs is zero because pixel outputs have random phase. Then

$$
\begin{array}{ll}
M_{i j}=C_{i j}=0 & \text { for } i \neq j \\
M_{i i} \rightarrow M_{i} & \text { (a real vector) } \\
C_{i i} \rightarrow C_{i} & \text { (a real vector) }
\end{array}
$$

$M$ and $C$ can be treated either as real diagonal matrices or vectors, depending on the context.

$$
Q_{i j k l}=0 \text { for } i \neq j \text { or } k \neq 1
$$

and thus the non zero real Qs simplify to

$$
Q_{i i j j} \rightarrow Q_{i j}
$$

and may be treated as a real symmetric matrix. The dimensionality of the covariance matrix $Q$ describing the process has been reduced from four to two. Also, complex pixel outputs are replaced by a set of real intensities $z=\left[z_{i}\right], i=1,2, \ldots, N$ where $Z_{i}=\left|E_{i}\right|^{2}$.

With these simplifying assumptions, one can show that the probability density for $z$ can be written as a multidimensional integral over real conditional probability densities.

$$
P(Z / M, Q)=\int \cdots \int P(Z / C) P(C / M, Q) d C
$$

where $P(Z / C)$ is simply a product of negative exponential distributions.

$$
P(Z / C)=\frac{1}{\prod_{i} c_{i}} \exp \left\{-\sum_{i} Z_{i} / C_{i}\right\}
$$

and

$$
\mathrm{P}(\mathrm{C} / \mathrm{M}, \mathrm{Q})=\frac{1}{\left[(2 \pi)^{\mathrm{N}}|Q|\right\rceil^{1 / 2}} \exp \left\{-\frac{1}{2}(\mathrm{C}-\mathrm{M})^{\mathrm{T}} \mathrm{Q}^{-1}(\mathrm{C}-\mathrm{M})\right\}
$$

$\mathrm{P}(\mathrm{Z} / \mathrm{C})$ will be recognized as a product of independent probability densities where each $Z_{j}$ is distributed as a chi-square distribution with two degrees of freedom. The second expression 
is a standard real multivariate Gaussian distribution. The relations (4-4) and (4-5) are now replaced by the relations

$$
\mathrm{C}_{\mathrm{i}}=<\mathrm{Z}_{\mathrm{i}}>
$$

and

$$
Q=\left[Q_{i j}\right]=\left\langle\left(C_{i}-M_{i}\right)\left(C_{j}-M_{j}\right)\right\rangle .
$$

where <> again stands for an average with respect to the respective probability densities.

The weakly non Gaussian assumption comes about by assuming, as in the earlier detection analysis, that $Q$ is small, so that $C<<M$ with high probability. The presence of the term in the integral involving $Q$ introduces correlated noise into the $Z_{i}$. As before, the signal manifests itself as a perturbation $M \rightarrow M+\delta M$, where $\delta M<M$. The perturbation $\delta M=\left[\delta M_{i}\right], i=1,2, \ldots N$, can be treated as a real vector with $\mathrm{N}$ components.

If one invokes the assumption of stationarity/homgeneity in background noise, $M_{i}$ must be invariant to the choice of $i$, and $Q_{i f}$ must depend only on $(i-j)$. Then one can set

$$
\begin{aligned}
M_{i} & =M_{0} \\
Q_{i j} & =Q_{i-j}
\end{aligned}
$$

for all $i, j$. These relations will be utilized later in the analysis.

Now calculate the first and second moments of $z_{i}$ from (4-9).

$$
\begin{aligned}
& \left.<\mathrm{Z}_{i}\right\rangle=\int \ldots \int \mathrm{Z}_{\mathrm{i}} \mathrm{P}(\mathrm{Z} / \mathrm{C}) \mathrm{P}(\mathrm{C} / \mathrm{M}, \mathrm{Q}) \mathrm{dCdZ} \\
& =\int \ldots \int \mathrm{Z}_{i} \mathrm{P}\left(\mathrm{Z}_{\mathrm{i}} / \mathrm{C}_{i}\right) d \mathrm{Z}_{i} \mathrm{P}(\mathrm{C} / \mathrm{M}, \mathrm{Q}) \mathrm{dC} \\
& =\int C_{i} P(C / M, Q) d C=M_{i} \\
& \left.<\mathrm{Z}_{i} \mathrm{Z}_{j}\right\rangle=\int \cdots \int \mathrm{Z}_{i} \mathrm{Z}_{j} \mathrm{P}(\mathrm{Z} / \mathrm{C}) \mathrm{P}(\mathrm{C} / \mathrm{M}, \mathrm{Q}) \mathrm{dCdZ} \\
& =\int \ldots \int Z_{i} Z_{j} P\left(Z_{i} / C_{i}\right) \dot{P}\left(Z_{j} / C_{j}\right) d Z_{i} d z_{j} P(C / M, Q) d C \quad(i \neq j)
\end{aligned}
$$




$$
\begin{array}{cc}
=\int \ldots \int \mathrm{Z}_{i}^{2} \mathrm{P}\left(\mathrm{Z}_{i} / \mathrm{C}_{i}\right) \mathrm{dz} \mathrm{Z}_{i} \mathrm{P}(\mathrm{C} / \mathrm{M}, \mathrm{Q}) \mathrm{dC} & (i=j) \\
=\int \cdots \int \mathrm{C}_{i} \mathrm{C}_{j}\left(1+\delta_{i j}\right) \mathrm{P}(\mathrm{C} / \mathrm{M}, \mathrm{Q}) \mathrm{dC} & (4-16) \\
=\left(Q_{i j}+\mathrm{M}_{i} \mathrm{M}_{j}\right)\left(1+\delta_{i j}\right) &
\end{array}
$$

One can solve for the $Q_{i j}$ in terms of the first and second moments of $Z_{i}$, which are measured quantities.

$$
\dot{Q}_{i j}=\frac{\left\langle Z_{i} Z_{j}\right\rangle}{1+\delta_{i j}}-M_{i} M_{j}
$$

By virtue of $(4-14)$ it is seen that the $z_{j}$ constitute a wide sense stationary process (see p. 302 of [17]), as expected.

\subsection{The Ideal Detection Statistic}

In order to derive an approximate form for the ideal detection statistic, let $C \rightarrow C+M$ in (4-9) and ignore the constant of proportionality.

$$
\mathrm{P}(\mathrm{Z} / \mathrm{M}, \mathrm{Q}) \sim \int \cdots \int \frac{1}{\prod_{i}\left(\mathrm{M}_{i}+\mathrm{C}_{i}\right)} \exp \left\{-\sum_{i} \mathrm{Z}_{i} /\left(\mathrm{M}_{i}+\mathrm{C}_{i}\right)-\frac{1}{2} \mathrm{C}^{\mathrm{T}} \mathrm{Q}^{-1} \mathrm{C}\right\} \mathrm{dC}
$$

For the weakly non Gauss $i$ an case, $Q$ is small, $C_{i}<\left\langle M_{i}\right.$, and $C_{i}$ is neglected in the integral. The terms involving $Z_{i}$ and $M_{j}$ can be pulled outside the integral and one has

$$
\mathrm{P}(\mathrm{Z} / \mathrm{M}, \mathrm{Q}) \sim \frac{1}{\prod_{i} M_{i}} \exp \left\{-\sum_{i} Z_{i} / M_{i}\right\}
$$

Recall (2-32) for an ideal statistic $\chi$.

$$
\begin{aligned}
\chi & =\ln L(\mathrm{Z})=\delta \ln \mathrm{P}(\mathrm{Z} / \mathrm{M}, \mathrm{Q}) \\
& =-\sum_{i} \frac{\delta \mathrm{M}_{i}}{\mathrm{M}_{i}}+\sum_{i} \frac{\mathrm{Z}_{i} \delta \mathrm{M}_{i}}{\mathrm{M}_{i}^{2}}
\end{aligned}
$$

The first term is a bias term which does not depend on $z_{j}$ and may therefore be ignored. Thus one can set

$$
\chi=\sum_{i} \frac{Z_{i} \delta M_{i}}{M_{i}^{2}}
$$


While the individual $Z_{j}$ are not Gaussian, one should note that a local average over $Z_{i}$ where $\delta M_{i}$ changes very 1 ittle, as would obtained with spatial-temporal filtering, will produce a quantity whose statistics approach Gaussian by virtue of the CLT.

\subsection{Derivation of the SNR}

The statistics of $\chi$ can now be used to calculate the generalized SNR using (2-7).

$$
\begin{gathered}
\left\langle\chi>_{N}=\sum_{i} \frac{\delta M_{i}}{M_{i}}=\operatorname{Tr}\left(\delta M^{-1}\right)\right. \\
<\chi>_{S N}=\sum_{i} \frac{\delta M_{i}}{M_{i}}+\sum_{i} \frac{\delta M_{i}^{2}}{M_{i}^{2}}=\operatorname{Tr}\left(\delta M^{-1}\right)+\operatorname{Tr}\left(\delta M^{-1} \delta M^{-1}\right) \\
<\chi>_{N}=\sum_{i} \sum_{j} \frac{\delta M_{i}}{M_{i}^{2}} \frac{\delta M_{j}}{M_{j}^{2}}<Z_{i} Z_{j}>=\sum_{i} \sum_{j} \frac{\delta M_{i}}{M_{i}^{2}} \frac{\delta M_{j}}{M_{j}^{2}}\left(Q_{i j}+M_{i} M_{j}\right)\left(1+\delta_{i j}\right) \\
=\sum_{i} \sum_{j} \frac{\delta M_{i}}{M_{i}^{2}} Q_{i j} \frac{\delta M_{j}}{M_{j}^{2}}+\sum_{i} \frac{\delta M_{i}}{M_{i}} \sum_{j} \frac{\delta M_{j}}{M_{j}}+\sum_{i} \frac{\delta M_{i}^{2}}{M_{i}^{4}} Q_{i i}+\sum_{i} \frac{\delta M_{i}^{2}}{M_{i}^{2}} \\
=\sum_{i} \sum_{j} \frac{\delta M_{i}}{M_{i}^{2}} Q_{i j} \frac{\delta M_{j}}{M_{j}^{2}}+\left(\operatorname{Tr}\left(\delta M^{-1}\right)\right)^{2}+\operatorname{Tr}\left(M^{-1} \delta M^{-1} Q^{-1} \delta M^{-1}\right) \\
+\operatorname{Tr}\left(\delta M^{-1} \delta M M^{-1}\right)
\end{gathered}
$$

where (4-16) has been used for $\left\langle Z_{i} Z_{j}\right\rangle$. When the trace expressions are employed, $M$ and $\delta M$ should be interpreted as $N \times N$ diagonal matrices. Insertion of these expressions into the SNR expression, $(2-7)$, yields, finally

$$
S N R=\frac{\left(\operatorname{Tr}\left(\delta M^{-1} \delta M M^{-1}\right)\right)^{2}}{\operatorname{Tr}\left(\delta M M^{-1} \delta M M^{-1}\right)+\sum_{i} \sum_{j} \delta M_{i} M_{i}^{-2} Q_{i j} M_{j}^{-2} \delta M_{j}+\operatorname{Tr}\left(M^{-1} \delta M M^{-1} Q^{-1} \delta M M^{-1}\right)}
$$

This expression has some interesting properties. It is analogous to the expression derived previously for the more general complex non Gaussian case [6]. In the limit where $Q$ is small (the Gaussian limit), the SNR becomes

$$
\operatorname{SNR}=\operatorname{Tr}\left(\delta M^{-1} \delta M^{-1}\right)
$$

Recalling (2-46), it is seen that this SNR expression is the same as for the real Gaussian case except that it is larger by a factor of two. The SNR is what one should expect for the random (or "speckle") noise limit. When $Q$ is not negligible, the other two terms in the denominator of the SNR expression, which are both positive, will cause a degradation of SNR. Even though $Q$ is 
smal1, it may be possible for these terms to dominate the first term, and thus provide a noise floor which is well above random (i.e., "speckle") noise. This situation would most likely occur where $Q_{i j}$ (which depends only on $i-j$ ) has spectral components (e.g., in $\bar{K}$ or $(K, \Omega)$ space) which are similar to that for the signal. A statistical analysis of ambient data (which can be perturbed to include the effect of a postulated signal) should reveal the extent to which non Gaussian noise degrades SNR.

From the stationarity/homogeneity assumption, (4-14), the matrix $M$ can be written

$$
M=M_{0} I_{N}
$$

where $I_{N}$, as before, is the $N \times N$ identity matrix. With this assumption the SNR expression (4-25) simplifies to

$$
S N R=\frac{\left(\operatorname{Tr}\left((\delta M)^{2}\right)\right)^{2}}{\left.M_{o}^{2} \operatorname{Tr}\left((\delta M)^{2}\right)\right)+\sum_{i} \sum_{j} \delta M_{i} Q_{i j} \delta M_{j}+\operatorname{Tr}(\delta M Q \delta M)}
$$

Whether $\delta M$ should be treated as a vector or a matrix in this equation can be seen from the context in which it is used. If one uses the stationarity/homogeneity condition for $Q$ in (4-25), the SNR expression further simplifies to

$$
S N R=\frac{\left(\operatorname{Tr}\left((\delta M)^{2}\right)\right)^{2}}{\left(M_{o}^{2}+Q_{o}\right) \operatorname{Tr}(\delta M)^{2}+\sum_{i} \sum_{j} \delta M_{i} Q_{i-j} \delta M_{j}}
$$




\section{An Approach to Handling Strongly Non Gaussian Statistics}

\subsection{Introduction}

The equations developed in section 3 are predicated on statistics which are at worst weakly non Gaussian. The statistical behavior of radar ocean images can be strongly non Gaussian, particularly at low grazing angle (LGA) when using $\mathrm{HH}$ polarization where the images are afflicted with stirong spikes. It has been noted that the general non Gaussian detection problem is intractable. The issue then is to find some method for reconciling this strongly non Gaussian behavior with the need to find tractable approaches to this problem.

A clue to an approach here comes from a recognition of the fact that the statistics of weak ambient current fields which underlie the radar images may be much better behaved and much more nearly Gaussian than the image itself. The signal perturbation which is manifested as a very weak surface current ends up producing breaking or sharply crested waves with a large radar signature, particularly at LGA. Thus, it may be argued that the underlying processes which may be nearly Gaussian end up producing a highly non Gaussian image by virtue of a) nonlinear hydrodynamics and b) the nonlinear functional relation, embodied in the scattering integral, relating the radar image to ocean surface height.

This view of the problem suggests that it may be possible to develop image processing or conditioning methods which will remove or compensate for this non Gaussian behavior to an extent where the analysis of section 4 can be applied. The complexity of the nonlinear hydrodynamics and scattering theory is so great that there is little prospect of developing a rigorous theory for completely "Gaussianizing" the radar images. However, this view does suggest that heuristic image conditioning techniques, based in part on measured ambient image statistics, might prove quite effective. An important component of the image conditioning would be the use of ZMNL functions whose purpose it would be to convert highly non Gaussian amplitude distributions (e.g., those with long tails caused by the presence of spikes) to nearly Gaussian distributions in the case of complex pixel outputs, or negative exponential distributions in the case of (non negative and real) intensities.

\subsection{Processing of Single Pixel Statistics}

In order to gain insight for the analysis to come, consider the case of a single pixel with intensity $z$.

\subsubsection{The Underlying Gaussian Case}

Consider first the case where the underlying statistics are Gaussian, which yields a probability density for $z$ which is 
Chi-square with two degrees-of-freedom, i.e., a negative exponential distribution. For the noise only case,

$$
\mathrm{P}(\mathrm{Z} / \mathrm{M})=\frac{1}{\mathrm{M}} \exp \{-\mathrm{Z} / \mathrm{M}\}
$$

where

$$
\mathrm{M}=\langle\mathrm{Z}>
$$

is the mean value of the intensity. This probability density can be considered to be a specialization of (4-10) to the one dimensional case. When signal is present, $M \rightarrow M+\delta M$, where $\delta M \ll M$ as before, and thus

$$
\mathrm{P}(\mathrm{Z} / \mathrm{M}+\delta \mathrm{M})=\frac{1}{\mathrm{M}+\delta \mathrm{M}} \exp \{-\mathrm{Z} /(\mathrm{M}+\delta \mathrm{M})\}
$$

An ideal statistic for detection is the LR, $L(Z)$.

$$
\begin{aligned}
L(Z)=\frac{P(Z / M+\delta M)}{P(Z / M)} & =\frac{M}{M+\delta M} \exp \{Z / M-Z /(M+\delta M)\} \\
& \cong 1+Z \delta M / M^{2}
\end{aligned}
$$

Since $L(Z)$ is an affine transformation on $Z$, one can use for the ideal statistic $\chi=Z$.

One can then readily calculate the quantities required for the generalized SNR.

$$
\begin{aligned}
<\chi>_{\mathrm{N}} & =\mathrm{M} \\
<\chi>_{\mathrm{SN}} & =\mathrm{M}+\delta \mathrm{M} \\
<\chi^{2}>_{\mathrm{N}} & =2 \mathrm{M}^{2}
\end{aligned}
$$

Inserting these results into the SNR expression, (2-7), yields

$$
\mathrm{SNR}=\frac{(\delta M)^{2}}{M^{2}}
$$

This result should come as no surprise since it can be recognized as a specialization of $(4-26)$ for SNR to the single pixel Gaussian case.

If one had used some nonlinear function of $z$ for $\chi$, e.g., $\chi=z^{2}$, one can show that the calculated SNR for the pixel could never be higher. 


\subsection{Pixel Statistics for LGA Sea clutter}

Extensive measurements have been made over the years of the probability distribution for the intensity of radar backscatter from the ocean surface. It is well known that these distributions depart from Gaussian, i.e. negative exponential in the intensity, and that this departure becomes very significant at LGA. The distribution broadens with decreasing cell size and grazing angle. The effect of this broadening is to increase the observed dynamic range between small and large intensities. The broadening is most pronounced for HH (Vs. VV, say) polarization where the return is observed to be very spiky.

Various models have been used to approximate these RCS fluctuations, including the log-normal distribution. However, the model of choice by many radar researchers (e.g., RSRE) is the "K" distribution [18-21]. The intensity distribution, $P(\mathrm{Z})$, for a'pixel output is written as a composite integral (referred to as the compound $\mathrm{K}$-distribution).

$$
P(Z)=\int_{0}^{\infty} P(Z / M) P(M) d M
$$

where

$$
\begin{aligned}
& P(Z / M)=\frac{1}{M} \exp \{-Z / M\} \\
& P(M)=\frac{b^{v}}{\Gamma(v)} M^{v-1} e^{-b M}
\end{aligned}
$$

The constant $b$ will be recognized as simply a scale factor in the $M$ distribution, while $v$ affects the shape of the distribution. $P(M)$, The Gamma distribution which enters into the Gamma function, will be recognized as a Chi-square distribution of $2 \mathrm{v}$ degrees of freedom. It acts as the spread function for the negative exponential function, $P(Z / M)$, sometimes referred to as the speckle function. $P(Z)$ can readily be written in terms of the function, $\mathrm{K}_{v} \mathrm{O}$, the modified Bessel function, and hence the term " $K$ " distribution. However, that expression will not be given here since it is not required in the analysis which follows. Ward, et. a]., [18], have performed statistical analyses of radar backscatter data to determine the dependence of $v$ on relevant parameters of interest. At $X$-band they quote the following expression for $v$,

$$
\log _{10} v=(2 / 3) \log _{10} \phi+(5 / 8) \log _{10} 1+\sigma-k
$$

where 


$$
\begin{aligned}
\phi & \equiv \text { grazing angle in degrees } \\
1 & \equiv \text { cross range resolution in meters } \\
\sigma & =-1 / 3 \text { for up/down swell radar pointing } \\
& =+1 / 3 \text { for cross swell radar pointing } \\
& =0 \text { for no swell or intermediate radar pointing } \\
k & =1 \text { for } V V \text { polarization } \\
& =1.7 \text { for HH polarization }
\end{aligned}
$$

A typical choice of parameters for LGA radar is $\phi=4$ degrees, $I=40 \mathrm{~m}, \sigma=0, k=1.7$ for $H H$, which yields $v \cong 0.5$. This value of $v$ will be used in a representative calculation later.

\section{2,3 The SNR Loss for K-Distributed sea Clutter}

Recall the SNR formula (5-6) which resulted from the perturbation of $M$ in the underlying Gaussian case. Consider now the SNR for $K$-distributed sea clutter where $P(Z / M)$ is perturbed in exactly the same manner.

$$
P(Z / M)=\frac{1}{M} e^{-Z / M} \rightarrow \frac{1}{B M} e^{-Z / B M}
$$

where

$$
\begin{array}{ll}
B=1 & \text { signal absent } \\
B=1+\delta M / M & \text { signal present }
\end{array}
$$

Eq. $(5-7)$ for $P(Z)$ is then

$$
P(Z)=\frac{1}{\Gamma(v)} \int_{0}^{\infty} \frac{1}{B M} \exp (-Z / B M) M^{v-1} \exp (-M) d N
$$

Here $\mathrm{b}$ has been set equal to one, since it is a fixed scale factor which will not affect SNR.

One can now evaluate the nth moment of $z,\left\langle z^{n}\right\rangle$, without having to evaluate $P(Z)$ itself. Recalling the definition of the Gamma function, $\Gamma(v)$,

$$
\Gamma(v)=\int_{0}^{\infty} x^{v-1} e^{-x} d x
$$


one can readily show

$$
\begin{aligned}
\left.<\mathrm{Z}^{\mathrm{n}}\right\rangle & \left.=\frac{1}{\Gamma(v)} \int_{0}^{\infty} \frac{1}{\mathrm{BM}} \int_{0}^{\infty} \mathrm{Z}^{\mathrm{n}} \exp (-\mathrm{Z} / \mathrm{BM})\right) \mathrm{aZM}^{v-1} \exp (-\mathrm{M}) \mathrm{dM} \\
& =\mathrm{B}^{\mathrm{n}_{n}} ! \Gamma(\mathrm{n}+v) / \Gamma(v)
\end{aligned}
$$
formula.

The required moments are readily deduced from the above

$$
\begin{aligned}
& <\mathrm{Z}>_{\mathrm{SN}}=(1+\delta \mathrm{M} / \mathrm{M}) \mathrm{v} \\
& <\mathrm{Z}>_{\mathrm{N}}=\mathrm{v} \\
& \left\langle\mathrm{Z}^{2}>_{\mathrm{N}}=2 \mathrm{v}(1+\mathrm{v})\right.
\end{aligned}
$$

The generalized SNR is then

$$
S N R=\left(\frac{v}{v+2}\right)\left(\frac{\delta M}{M}\right)^{2}
$$

The only change from the previous expression, (5-6), is the factor involving $v$ which produces a decrease in SNR. For the example noted above where $v \cong 0.5$, the degradation in SNR is $7 \mathrm{~dB}$, a significant loss for many applications. This single pixel loss in SNR will, of course, be reflected directly in a loss in SNR for an image. In the limit of weak signal and statistically independent pixels the loss in SNR for the image will also be 7 $\mathrm{dB}$.

\subsection{Restoration of the SNR Loss}

Is. this loss in detection performance irretrievable? The answer is no, as will now be demonstrated.

The loss in SNR comes about because $Z$ is no longer an ideal detection statistic, i.e., a monotonic function of the LR based on the expression for $P(Z)$. A derivation of a closed-form expression for this ZMNL function of $Z$ appears to be intractable, however. Nevertheless, it is sufficient to recognize that the distribution $P(Z / M)$ can be recovered from $P(Z)$ by replacing $Z$ with an appropriate numerically derived ZMNL function of $Z$, as will be shown in the next section. Because the function is monotonic, the process is invertable and involves no loss of information.

In summary, then, the procedure requires that $z$ be replaced by a new $Z$ which is a ZMNL function of the old $Z$, with a negative exponential probability density $P(Z / M)$, and this new $Z$ is used as the detection statistic. The SNR will be given by the original 
expression, (5-6), for the underlying Gaussian process. For the example cited, the $7 \mathrm{~dB}$ loss in SNR will be regained.

One must be careful to apply this technique only to the raw pixel intensities (except for spatial/spectral detrending) which have not been spatially and/or temporally averaged. Such averaging will, by virtue of the CLT, tend to Gaussianize the process and thereby reduce the potential improvement in SNR.

On the other hand, the presence of spurious amplitude modulations in the data, e.g., due to surface wave features such as wave groups or strongly directional swell waves, can significantly distort. the amplitude statistics. This distortion may in turn degrade or negate the performance gain which might otherwise be obtained with the use of a ZMNL function to produce the desired negative exponential statistics. If this interference involves wavelengths or directions which differ from the desired signal, one can employ a two-dimensional detrending filter (addressed elsewhere [22-24]) to attenuate/mitigate this interference. The detrended pixel outputs now can be employed to numerically calculate the desired ZMNL function which will be applied to these pixel outputs to maximize SNR.

\subsection{Transformation of Probability Distributions}

Let $x$ be a random variable which takes on positive real values, like a pixel intensity. Let $P(x)$ be the probability density function for $x$. The cumulative distribution function, denoted $F(x)$, is given by

$$
F(x)=\int_{0}^{x} P(u) d u
$$

$F(x)$ is a function which increases monotonically with $x$ from zero to one.

Consider a ZMNL function of $x$, denoted $Y(x)$, which will yield a probability density $Q(Y)$ whose form is specified. By the laws of probability

$$
Q(Y) d Y=P(X) d x
$$

This equation could be solved numerically to find $Y(X)$. However, there are some cases of special interest where $Y(X)$ can be obtained simply. The first is where $Q(Y)=1$ over the interval $(0,1)$. Choose $Y(X)$ so that $Y(0)=0$. Then

$$
Y=\int_{0}^{x} P(u) d u=F(x)
$$

A second example, the case of interest, is the choice 


$$
Q(Y)=e^{-Y}, \quad Y \geq 0
$$

Then

$$
\int_{0}^{x} e^{-s} d s=\int_{0}^{x} P(u) d u=F(x)
$$

from which, it follows

$$
Y(x)=-\ln (1-F(x))
$$

Note that $Y$ is a monotonically increasing function of $x$ and thus one can invert this relation to determine $x(Y)$.

$P(x)$, and thus $F(x)$, cam be obtained numerically by statistical analys is of data. In some situations it may be desirable to approximate $F(x)$ by some function such as a polynomial. For the problem of interest, the desired functional replacement is

$$
\mathrm{Z} \rightarrow-\operatorname{In}(1-\mathrm{F}(\mathrm{Z}))
$$

Finally, it should be observed that if the pixel intensity $z$. is replaced by any ZMNL monotonic function of $Z$, the above procedure still yields a random variable whose probability density is the same negative exponential. Thus, for example, $Z$ can be replaced by any power of $Z$ since this operation will not affect the result. In particular, $z$ can be replaced by $z^{1 / 2}$, which is the modulus of the complex pixel value. The choice of whether to use $Z$ or some other monotonic function of $Z$ prior to the replacement of $(5-23)$ is simply a matter of computational convenience. 
6. Implications for Processing Improvements and Further Analysis

\subsection{Introduction}

Due to limitations in time and effort, detailed theoretical analysis of the non Gaussian detection problem will not be addressed further here. Additional analysis has been performed, and may be the subject of future documentation. One reasonable objective of future analysis would be to obtain an efficient processing methodology which would reduce the detection problem to a classical form, i.e., an additive signal in white Gaussian noise.

Actual image data frequently contains artifacts due to equipment problems, scattering/hydro phenomenology or ambient background which causes the statistics to depart significantly from the non Gaussian noise models considered earlier. It has been seen that the appropriate use of ZMNL functions may be able to mitigate the effect of strongly non Gaussian behavior. However, the utility of this mitigation procedure may be seriously degraded by these uncompensated data artifacts.

The next two sections provide a qualitative description of methods for reducing data artifacts. It should not be difficult to restate these methods in terms of clearly defined theoretical formulations of the required algorithms.

For the purpose of the discussion to follow, the problem is discussed in terms of spatial coordinates $(x, y)$ and transform coordinates $\bar{K}=\left(K_{x}, K_{y}\right)$. In the case of a fixed (e.g., hillside) LGA RAR, the coordinates of interest would, of course, be $(y, t)$ and $(R, \Omega)$.

\subsection{Two-Dimensional Spatial Detrending}

The image data may contain gross changes in intensity due to antenna pattern variations, wind patterns, swell waves, wave groups or other phenomena. These can be distinguished from the signals of interest because their spatial (or temporal) frequencies are different. Spatial detrending to remove or reduce this type of noise can be thought of as a spatial whitening operation. A number of detrending methodologies have been employed, unfortunately with mixed results. One must be careful to avoid approaches which produce marginal or unsatisfactory results from the standpoint of performance and computation speed. It is particularly important to choose the parameters of the detrender so that it does not significantly attenuate signals of interest.

The spatial detrender is fundamentally different than a spatial filter because it acts to normalize out the undesired interference, a process involving division, which leaves the number of pixels in the image unchanged. Spatial filters involve 
area averaging which attempts to average out undesired artifacts and may reduce the number of pixels.

The spatial detrender should have the following attributes:

- Operation in two dimensions.

- No (significant) attenuation of the signal

- Use fast processing algorithms, e.g.,

fast convolution using the FFT.

The image intensity function to be detrended is denoted $z(x, y)$. Corresponding variables in the transform domain are denoted $\tilde{Z}(\bar{K})$. The main steps in the detrending process are indicated below:

a. Select a subimage of $z(x, y)$ for spatial detrending. The size of this subimage is limited by practical data processing considerations.

b. Zero pad the resulting $z(x, y)$ in both $x$ and $y$ by adding zeros around the edges. This procedure will usually quadruple the image area.

$c$ : Select a low pass filter function $W(x, y)$, which is real, positive, symmetric about and centered at the origin. $W(x, y)$ is contoured to pass low spatial frequencies but reject spatial frequencies corresponding to signal of interest.

d. Construct the first order detrending function $c(x, y)$ by taking the two-dimensional convolution,

$$
C(x, y)=W(x, y) \otimes z(x, y)
$$

This operation is efficiently performed in transform space by multiplying

$$
\tilde{\mathrm{C}}(\overline{\mathrm{K}})=\tilde{W}(\overline{\mathrm{K}}) \tilde{\mathrm{Z}}(\overline{\mathrm{K}})
$$

and taking the inverse two-dimensional FFT. The nature of the functions $W$ and $Z$ ensures that $c(x, y)$ will be non negative and real.

e. $c(x ; y)$ must be corrected for droop due to edge effects. The correction function $D(x, y)$ is found by convoluting $W(x, y)$ with a rectangle function, $R(x, y)$.

$$
\mathrm{D}(\mathrm{x}, \mathrm{y})=\mathrm{W}(\mathrm{x}, \mathrm{y}) \otimes \mathrm{R}(\mathrm{x}, \mathrm{y})
$$

Since $D(x, y)$ does not depend on $Z(x, y)$, it only has to be computed once and stored. The compensated detrending function is 


$$
\mathrm{C}_{\mathrm{o}}(\mathrm{x}, \mathrm{y})=\mathrm{C}(\mathrm{x}, \mathrm{y}) / \mathrm{D}(\mathrm{x}, \mathrm{y})
$$

This function only needs to be computed for values of $x$ and $y$ within the original subimage.

$f$. The subimage $z(x, y)$ is now whitened by computing $z(x, y) / c_{0}(x, y)$. The detrended subimages are then reassembled to form a complete detrended image.

The spatial detrender may be effective on the long wavelength phenomena, but may be ineffective on shorter wavelength noise such as surface waves and wave groups. For these one can use spectral detrending, discussed next.

\section{6,3 Two-Dimensional Spectral Detrending}

Spectral detrending can be thought of as a spectral whitening filter. The steps required are quite similar to those outlined above for spatial whitening. The spectral detrending operation should immediately follow spatial detrending. The steps in the process are outlined below:

a. Select a subimage of the intensity image, $z(x, y)$, for spectral detrending. Its size should be large, but the size may be 1 imited by practical data processing considerations.

b. Compute $\overline{\mathrm{z}}(\overline{\mathrm{K}})$ using a two-dimensional digital FFT. $\overline{\mathrm{Z}}(\overline{\mathrm{K}})$ is generally complex, so replace it by $|\tilde{z}(\overline{\mathrm{K}})|^{2}$.

c. Zero pad $|\tilde{z}(\bar{K})|^{2}$ in $K_{X}$ and $K_{Y}$. This will usually double the image size in $K_{x}$ and $K_{y}$.

d: Choose a lowpass filter function $W(\bar{K})$ which is_real, non negative, symmetric about and centered on the origin, $\bar{k}=0$. The rate at which $W(\bar{K})$ falls off with increasing $K$ (decreasing wavelength) needs to be determined. One reasonable estimate might be that $W(\bar{K})$ should taper to a small value at $K \cong 2 \pi / 100$ ( 100 meter wavelength).

\section{e. Compute the spectral detrending function}

$$
C(\overline{\mathrm{K}})=\left[W(\overline{\mathrm{K}}) \otimes|\tilde{\mathrm{Z}}(\overline{\mathrm{K}})|^{2}\right]^{\frac{1}{2}}
$$

The nature of the functions $w(\bar{K})$ and $|\tilde{z}(\tilde{K})|^{2}$ will ensure that the two-dimensional convolution will be non negative and real. The convolution is efficiently performed in $(x, y)$ space by taking the 
inverse FFTs of $W(\bar{K})$ and $|\tilde{\mathrm{Z}}(\overline{\mathrm{K}})|^{2}$, multiplying the functions and then taking an FFT to obtain the desired K-space convolution. ( 1 t should be noted that the inverse transform of $|\vec{z}(\bar{K})|^{2}$ is the spatial autocorrelation of $z(x, y)$. A careful examination of this autocorrelation function may be useful for uncovering deficiencies in the data processing).

f. $C(\overline{\mathrm{K}})$ must be corrected for droop due to edge effects. The correction function $D(\bar{K})$ is found by convolving $W(\bar{K})$ with a rectangle function $R(\overline{\mathrm{K}})$.

$$
D(\bar{K})=W(\bar{K}) \otimes R(\bar{K})
$$

Since $D(\bar{K})$ does not depend on $\bar{Z}(\bar{K})$, it only has to be computed once and stored. The compensated detrending function is

$$
C_{o}(\bar{K})=C(\bar{K}) / D(\bar{K})
$$

This function only needs to be computed for values of $\overline{\mathrm{K}}$ in the non zero-padded region.

g. The function $\overline{\mathrm{Z}}(\overline{\mathrm{K}})$ is now whitened by computing

$$
\tilde{\mathrm{Z}}(\overline{\mathrm{K}}) / \mathrm{C}_{\mathrm{o}}(\overline{\mathrm{K}})
$$

h. The result can now be transformed back to compute a new spatial subimage $z(x, y)$. The various subimages, each spectrally whitened in this manner, can be reassembled into a complete image.

The spectral whitening procedure outlined above should be effective in suppressing wave group noise, and harmonics thereof, a phenomenon which strongly afflicts LGA RAR images. In high grazing angle SAR images one would expect that gravity-swell wave noise could be more prominent, and the shape of $W(\bar{K})$ should be chosen to suppress these.

\subsection{Additional Processing Steps}

After spatial and spectral detrending have been performed, an appropriate ZMNL function can be applied which should more effectively eliminate the strongly non Gaussian character of the data. To the extent that this procedure has been successful, one can employ the composite probability integral (4-9) for $P(Z / M, Q)$. A potential further benefit is that the $Q$ matrix of $(4-14)$ is approximately a scalar due to the flattened spectrum.

Recalling (4-21) for the ideal detection statistic and the stationarity condition $M_{j}=M_{0}$ of $(4-14)$, one has for the ideal detection statistic, 


$$
\chi=\frac{1}{\mathrm{M}_{\mathrm{o}}^{2}} \sum_{\mathrm{i}} \mathrm{Z}_{\mathrm{i}} \delta \mathrm{M}_{\mathrm{i}}
$$

where each $Z_{i}$ has a negative exponential probability density according to $(4-19)$. If $\delta M_{i}$ is very slowly varying on the scale of a pixel dimension, one can perform a local average over the $z_{i}$ without significantly compromising the computation of $\chi$. This local averaging can be implemented with spatial filtering (performed efficiently in $\mathrm{K}$-space, as usual). The new detection statistic takes the form

$$
\chi=\frac{1}{M_{o}^{2}} \sum_{j} z_{j} \delta M_{j}
$$

where each $z_{j}$ is obtained as a local average over a significant number of pixels. The $\left\langle z_{j}\right\rangle$ is a constant which is a bias term in $\chi$, which is of no consequence. However, the probability density for each $Z_{j}$ approaches Gaussian by virtue of the CLT. To the extent that these approximations are valid, the detection problem (based on the $Z_{j}$ ) has been reduced to the classical problem of additive signal in white Gaussian noise. The above detection statistic entails the correlation of the received data samples with the expected signal (so-called "correlation reception").

The above somewhat heuristic discussion suggests the general approach which could be used in the development of a more rigorous treatment of the problem. Logical extensions of this theory would include multichannel detection (e.g., frequency, polarization and aperture). 


\section{GLOSSARY OF SYMBOLS AND ACRONYMS}

$C=$ dummy moment matrix or vector

$E=$ complex pixel output, matrix or vector

$F=$ cumulative probability distribution function

$I_{N}=N \times N$ identity matrix

$i, j, k, l=$ space-time indices

$\overline{\mathrm{K}}=\left(\mathrm{K}_{x}, \mathrm{~K}_{\mathrm{y}}\right)=$ spatial wavenumber vector

$L(Z)=$ Likel ihood ratio function of $Z$

$N=$ number of space-time cells

$M=$ moment matrix of intensities

$P()=$ probability density function

$P_{D}=$ probability of detection

$P_{F}=$ probability of false al arm

$Q=$ moment matrix (2nd or 4 th order)

, $W()=$ weighting function

$x, y=$ spatial coordinates

$Z=$ scalar/vector/matrix pixel intensity

CLT = Central Limit Theorem

$F F T=$ Fast Fourier Transform

LGA $=$ low grazing angle

$L R=$ Likel ihood ratio

RAR = real aperture radar

ROC $=$ receiver operating characteristic

SAR = synthetic aperture radar

$S N R=$ signal to noise ratio

$Z M N L=$ zero memory nonl inear

$\Gamma=$ Gamma function

$\Omega=$ radian frequency

$\chi=$ ideal detection statistic

\langle\rangle$=$ expectation or ensemble average

$\operatorname{Tr}()=$ trace of a matrix

$1 \mid=$ determinant of a matrix

[ ] = matrix of elements

$T=$ a superscript denoting transpose

$t=a$ superscript denoting Hermitian conjugate 


\section{REFERENCES}

1. Helstrom, C.W., "Statistical Theory of signal Detection," (Permagon Press, 1968).

2. Van Trees, "Detection, Estimation and Modulation Theory," Vols. I, II, III (J. Wiley and Sons; 1971).

3. Whalen, A.D., "Detection of Signal in Noise," (Academic Press, N.Y., 1971).

4. DiFranco, J.V., Rubin, W.L., "Radar Detection," (Prentice-Hall, 1968).

5. Manasse, R. "A Simplified Detection Theory Model Using Radar Maps," RDA-Logicon report, RDA-TR-104605-002, March 1977.

6. Manasse, R., "Detection of Random Signals in Weakly Non Gaussian Noise," Swerling, Manasse and Smith, March 1, 1990.

7. Poor, H.V., "An Introduction to Signal Detection and Estimation;" (Springer-Verlag, 2nd Ed. 1994).

8. Birkhoff, G., MacLane, S., "A Survey of Modern Algebra," (Macmitlan Co., N.Y., 1948).

9. Cramer, H., "Mathematical Methods of Statistics," (Princeton University Press, Sixth Ed., 1954).

10. Manasse, R., "The Use of Polarimetry for Enhancing the Detectability of Ocean Surface Features with Radar" LLNL Report UCRL-ID-118532, Sept. 1, 1994

11. Middleton, D., "Threshold Detection of Radar signals off the SEA Surface in Non-Gaussian Clutter and Deterministic Interference," 1. Coherent Detection. August, 1995, LLNL-MI-123025 (Preliminary).

12. Bellman, R., "Introduction to Matrix Analysis," (MCGraw-Hi11, 1960).

13. Novak, L.M., et. al., "Optimal polarimetric Synthetic-Aperture Radar Imagery," The Lincoln Laboratory Journal, Vol. 3, No. 2, 1990.

14. Novak, L.M., Bur 1, M.C., "Optimum Speckle Reduction in Polarimetric SAR Imagery," IEEE Trans. AES, Vol. 26, No. 2, March, 1990.

15. Novak, L.M., et. al., "Optimal Polarimetric Processing for Enhanced Target Detection," IEEE Trans. AES, Vol. 29, No. 1, Jan. 1993. 
16. Personal communications, Drs. Dennis Holliday (RDA-Logicon) and James Brase (LLNL).

17. Papoulis, A., "Probability, Random Variables and Stochastic Processes," (McGraw-Hill, 1965).

18. Ward, K.D., et. al., "Maritime Surveillance Radar, Pt. I Radar Scattering from the Ocean Surface, Pt. 11, Detection Performance Prediction in sea clutter," IEE Proceedings, Vol. 137, Pt. F, No. 2, April 1990 (and references contained therein).

19. Ward, K.D., "A Radar sea Clutter Model and its Application to Performance Assessment," IEE Conf. Publ. 216, Radar-82, 1982.

20. Baker, C.J., Ward, K.D., Watts, S., "The significance and Scope of the Compound K-distribution Model for Sea Clutter," IEE Conf. Publ. 281, Radar-87, 1987.

21. Personal communication, Dr. Robert Tough.

22. Manasse, R., memorandum to D. Hoiliday, "Observations on Detrending and Processing of Ocean Images," June 11, 1993.

23. Manasse, R., memorandum to J. Brase, "A proposed Signal Processing Methodology for RAR Data," Oct. 16, 1994.

24. Manasse, R., memorandum to J. Brase, "A Discussion of Some Image Processing and Detection Issues," Nov. 2, 1994. 